\title{
Dural Carotid-cavernous Fistula Mimicking Thyroid Orbitopathy
}

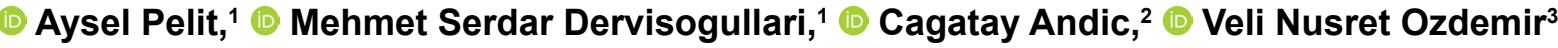 \\ ${ }^{1}$ Department of Ophthalmology Adana Clinic and Research Center, Başkent University Faculty of Medicine, Adana, Turkey \\ ${ }^{2}$ Department of Interventional Radiology Adana Clinic and Research Center, Başkent University Faculty of Medicine, Adana, Turkey \\ ${ }^{3}$ Department of Ophthalmology, Private Practice, Adana, Turkey
}

\begin{abstract}
In this study, we aim to present a case with dural carotid-cavernous fistula mimicking thyroid orbitopathy. The differential diagnosis of red-eye is very complex, and the caregiver should distinguish between all diagnoses.

Keywords: Carotid-cavernous fistula, thyroid orbitopathy, strabismus.
\end{abstract}

\section{Introduction}

The pathological communicative shunt between the carotid arterial system and the cavernous sinus is called carotid-cavernous fistula fistula (CCF). CCF can be classified as follows: direct or indirect, spontaneous or traumatic, with high or low pressure. According to the Barrow classification, Type $A$ is the direct communication between the intracavernous segment of the internal carotid artery (ICA) and cavernous sinus (CS). Type $B$ is the dural shunt between intracavernous branches of the ICA and CS. Type $C$ is the dural shunt between meningeal branches of the external carotid artery (ECA) and CS. Type D is a combined type of dural ICA and ECA shunt and CS ( $B+C$ type) (I).

The clinical findings of CCF are reduction of visual acuity, conjunctival chemosis, exposure keratopathy, eyelid edema, headache, orbital bruit, pulsating exophthalmos, proptosis, external ophthalmoplegia, central retinal vein occlusion (CRVO), branch retinal vein occlusion (BRVO), vitreous and/or retinal hemorrhage, vascular tortuosity, proliferative retinopathy, edema of the optic disc, atrophy of the optic nerve and secondary glaucoma. Diagnosis is based on a clinical ophthalmological examination, B-mode orbital echography, CT, MRI, MRI Angiography and intra-arterial digital subtraction angiography (DSA).

Differential diagnosis includes thyroid ophthalmopathy, orbital tumor and orbital pseudotumor, IgG4-Related orbitopathy, orbital hemorrhage, myasthenia gravis and cavernous sinus pathologies (sinus tumor, thrombosis, inflammatory lesions). Medical therapy of CCF includes artificial tears and antiglaucoma medications. Surgical treatment includes transcranial exploration of the cavernous sinus with a ligature of the ICA proximal and distal to the fistula, endovascular treatment by applying a thin platinum coil, fiber embolization coiling, balloon embolization or stent for aneurysm with wide neck or clamping of an aneurysm in its neck. Commonest complications in the eye are CRVO, atrophy of the optic nerve and secondary glaucoma (2).

Address for correspondence: Mehmet Serdar Dervişoğlu, MD. Baskent Universitesi Tip Fakultesi, Oftalmoloji Anabilim Dali Adana Klinik ve Arastirma Merkezi, Adana, Turkey

Phone: +90 5327720616 E-mail: serdarderv@hotmail.com

Submitted Date: December 21, 2018 Accepted Date: July 28, 2019 Available Online Date: December 27, 2019

${ }^{\circ}$ Copyright 2019 by Beyoglu Eye Training and Research Hospital - Available online at www.beyoglueye.com OPEN ACCESS This work is licensed under a Creative Commons Attribution-NonCommercial 4.0 International License. 


\section{Case Report}

Sixty-two-year-old female patient came to our clinic complaining of strain, redness, enlargement in the left eye and diplopia for six weeks. The patient's consent was obtained for this study. She had pterygium surgery in the left eye six weeks ago. There was no trauma in the history. She had diabetes melllitus and hypertension under control with medication. Her sister had thyroid pathology. Four weeks ago, in another clinic, TSH,T3,T4 were measured normal and according to the orbita MRI report (we could not obtain the images), there were mild proptosis, increase in retroorbital fat signal and enlargement in medial and lateral rectus muscles in the left eye compatible with thyroid orbitopathy. At that clinic, $60 \mathrm{mg} /$ day oral prednisolone was started for thyroid ophthalmopathy, and she was using $8 \mathrm{mg} /$ day for the day she came to our clinic.

In ophthalmological examination, best-corrected visual acuity (BCVA) was 0.8 (with+3.00-I.50@85) and 0.8 (+4.00-2.00@70) in right and left eyes, respectively. IOP values were $14 \mathrm{mmHg}$ in the right eye and $15 \mathrm{mmHg}$ in the left eye measured with Goldmann applanation tonometer. In slit-lamp, there was edema in the upper eyelid and conjunctiva of the left eye and Grade I nuclear sclerotic cataract bilaterally. In fundoscopy, there was mild retinal venous congestion. Although the primary position was ortophoric, abduction and adduction was deficient in the left eye (Fig. I). Direct and indirect light reflexes were normal. Hertel exophthalmometry measurements were 21 and 29 $\mathrm{mm}$ in the right and left eyes, respectively. The patient was consulted the endocrinology department. TSH was 0.9,T3 2.72, T4 was 0.68, AntiTG was 0.5, Anti TPO was 0 and TRAB was negative. The endocrinology council agreed on normal thyroid function, finally. In orbita MRI, there was an increase in retroorbital fat signal, enlargement in medial and lateral rectus muscles, and asymmetrical enlargement of the cavernous sinus in the left eye (Fig. 2) compatible with orbital pseudotumor. Although the superior ophthalmic vein enlargement is the mainstay of diagnosis of CCF in orbital MR, it was not obvious in our patient. Depending on our ongoing clinical suspicion, the patient was sent for cerebral MR angiography, which was reported as normal by the radiology department. The Radiology department was asked for a selective cerebral angiography option. The selective cerebral angiography indicated a low flow Type D dural CCF with rete type multiple feeders from the left middle meningeal artery, internal maxillary artery and left internal carotid artery (Fig. 3). The patient was treated with the combination of a selective particle (PVA) and coil embolization in two separate sessions from external carotid artery feeders (Fig. 4). Clinical and MRI findings also regressed after treatment (Fig. 5).

\section{Discussion}

The differential diagnosis of red-eye is very complex, and the caregiver must be comfortable with all diagnoses and be able to distinguish between them (3). Principally, the red
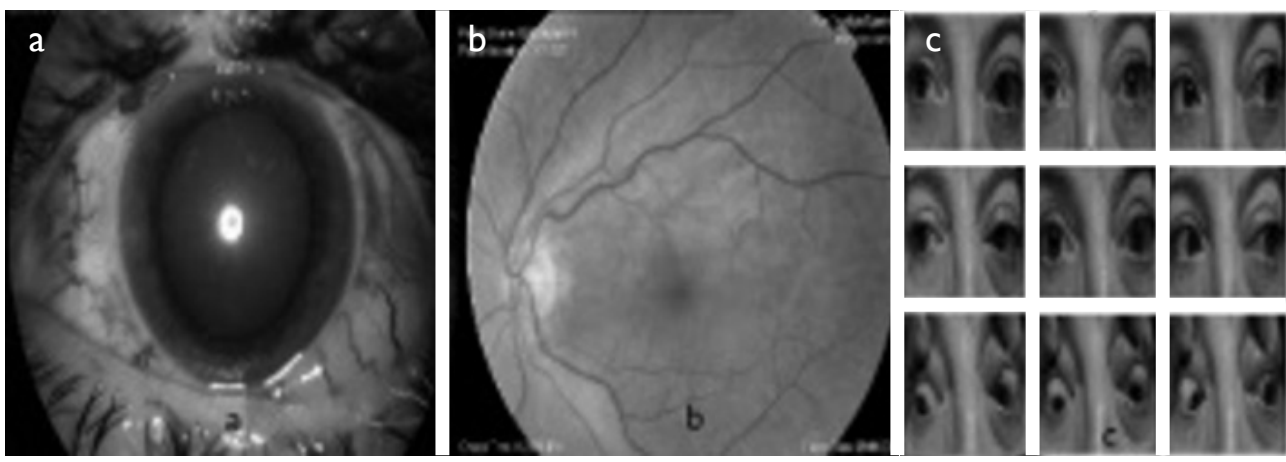

Figure I. (a) edema in the upper eyelid and conjunctiva of the left eye (b) fundoscopy and (c) the primary position was ortophoric, abduction and adduction were deficient in the left eye.
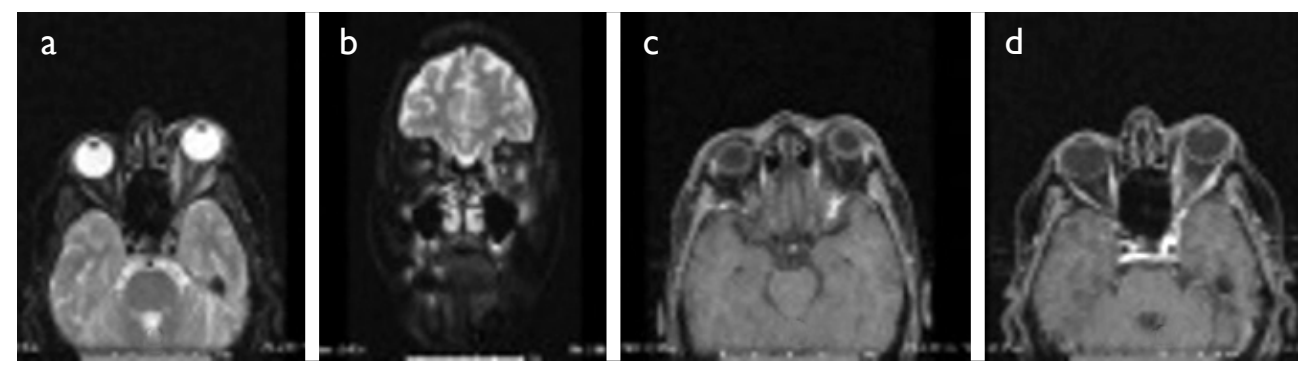

Figure 2. MRI findings in different sections. 


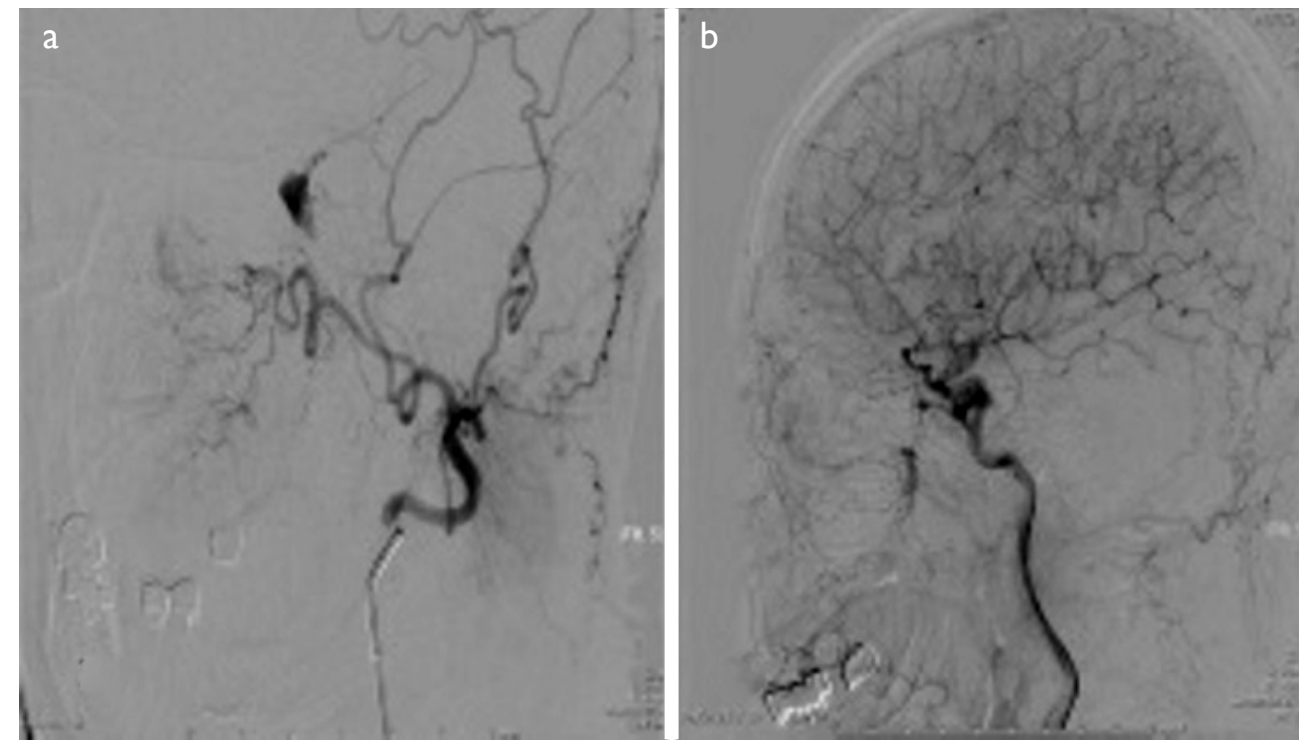

Figure 3. Selective cerebral angiography shows rete type multiple feeders from (a) left internal maxillary, middle meningeal artery and (b) left internal carotid artery.
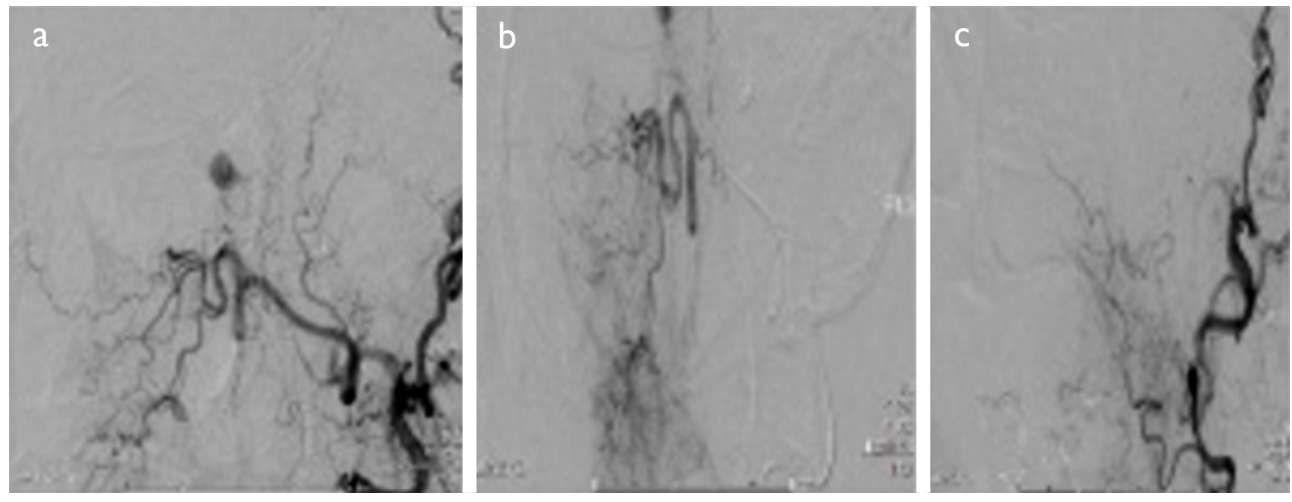

Figure 4. Endovascular treatment of the fistula with the combination of the particle (PVA) and coil embolization from externally carotid artery feeders respectively in a, b and c.
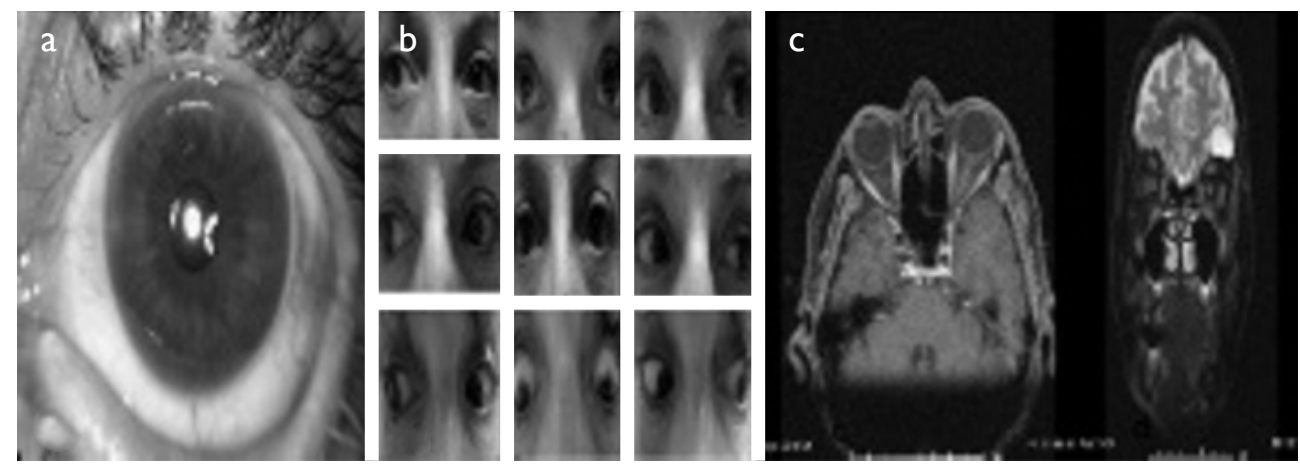

Figure 5. Regression in clinical $(\mathbf{a}, \mathbf{b})$ and $M R I(\mathbf{c})$ findings after treatment.

eye is the cardinal sign of ocular inflammation. The condition is usually benign and conjunctivitis is the most common cause of red eye, but the diagnosis of some other rare causes of red eye may be critical for management. Besides clinical findings, laboratory and imaging technologies help us for the differential diagnosis, but 'clinical sense' leads them all.
CCF can be an important cause of red eye and easily masquerade conjunctivitis. Xia et al. (4) presented a-73-year-old woman with a complaint of a red eye with gradual proptosis and mild restricted movement of the right eye who was misdiagnosed with conjunctivitis for two months. Computed tomography scan of the orbit of their patient showed an en- 
larged superior ophthalmic vein (SOV) and made their CCF diagnosis easier. Khan et al. (5) presented a 66-year-old patient who was treated previously as a case of conjunctivitis. They concluded that carotid-cavernous fistulas are a threat to the vision if left untreated due to delayed diagnosis and recommended considering bilateral carotid-cavernous fistula as a differential diagnosis in patients with an ongoing history of red eyes or those unresponsive to conventional topical treatment for conjunctivitis like symptoms. Campbell et al. also presented a CCF case masquerading conjuntivitis and sinusitis (6).

There was extraocular muscle enlargement (EOME) in orbital MR of our patient. Thyroid eye disease is the main cause of EOME, but non-thyroid related EOME mostly arises from neoplasias (7). Because of witnessing a red eye together with EOME in MRI and normal thyroid functions, in the light of our ophthalmologic examination, a vascular pathology was considered and MR angiography and selective cerebral angiography were asked from the radiology department. CCF may be classified as direct or dural. Direct CCFs are mostly traumatic in origin. Causes of dural CCFs include hypertension, fibromuscular dysplasia; Ehlers Danlos type IV and dissection of the ICA (8). In our patient, there was a low-pressure Type $D$ dural CCF according to the result of the selective cerebral angiography. There was no trauma in history, but she had hypertension. The patient was treated with endovascular coil embolization, and all findings regressed after treatment. Modern endovascular techniques offer the ability to successfully treat CCFs.

In the light of clinical findings, clinician should lead the laboratory and imaging technologies for the differential diagnosis in complex red eye cases.

\section{Disclosures}

Informed consent: Written informed consent was obtained from the patient for the publication of the case report and the accompanying images.

Peer-review: Externally peer-reviewed.

Conflict of Interest: None declared.

Authorship Contributions: Involved in design and conduct of the study (AP); preparation and review of the study (MSD); data collection (VNO, CA).

\section{References}

I. Thinda S, Melson MR, Kuchtey RW. Worsening angle closure glaucoma and choroidal detachments subsequent to closure of a carotid cavernous fistula. BMC Ophthalmol 20I2; I2:28. [CrossRef]

2. Scott IU. Carotid-Cavernous Fistula (CCF) Differential Diagnoses. Available from: http:// emedicine.medscape.com/ article/I2I7766-differential.

3. Graham RH. Red Eye Diferential Diagnosis. Available from: http:// emedicine.medscape.com/ article/I 192122-differential.

4. Xia Y. A red eye induced by a spontaneous carotid cavernous fistula. Am J Emerg Med. 2018;36:2336.el-2336.e2.

5. Khan S, Gibbon C, Johns S. A rare case of bilateral spontaneous indirect caroticocavernous fistula treated previously as a case of conjunctivitis. Ther Adv Ophthalmol 2018; 10:25I 584I418788303. [CrossRef]

6. Campbell S, Hack E, Philip B. A rare red eye: cavernous sinus dural arteriovenous fistula masquerading as conjunctivitis and sinusitis. Med J Aust 2019;210:398-399.el. [CrossRef]

7. Shafi F, Mathewson P, Mehta P, Ahluwalia HS. The enlarged extraocular muscle:to relax, reflect or refer? Eye (Lond) 20I7;31:537-44. [CrossRef]

8. Henderson AD, Miller NR. Carotid-cavernous fistula: current concepts in aetiology, investigation and management. Eye (Lond) 2018;32:164-72. [CrossRef] 